\title{
Ionospheric tomography using GNSS: multiplicative algebraic reconstruction technique applied to the area of Brazil
}

\author{
Fabricio dos Santos Prol $^{1} \cdot$ Paulo de Oliveira Camargo ${ }^{1}$
}

Received: 25 March 2015/Accepted: 8 September 2015/Published online: 22 September 2015

(c) Springer-Verlag Berlin Heidelberg 2015

\begin{abstract}
Experimental analysis was performed using multiplicative algebraic reconstruction technique (MART) to map the ionosphere over Brazil. Code and phase observations from the global navigation satellite system (GNSS) together with the international reference ionosphere (IRI) enabled the estimation of ionospheric profiles and total electron content (TEC) over the entire region. Twenty-four days of data collected from existing groundbased GNSS receivers during the recent solar maximum period were used to analyze the performance of the MART algorithm. The results were compared with four ionosondes. It was demonstrated that MART estimated the electron density peak with the same degree of accuracy as the IRI model in regions with appropriate geometrical coverage by GNSS receivers for tomographic reconstruction. In addition, the slant TEC, as estimated with MART, presented lower root-mean-square error than the TEC calculated by ionospheric maps available from the International GNSS Service (IGS). Furthermore, the daily variations of the ionosphere were better represented with the algebraic techniques, compared to the IRI model and IGS maps, enabling a correlation of the elevation of the ionosphere at higher altitudes with the equatorial ionization anomaly intensification. The tomographic representations also enabled the detection of high vertical gradients at the same instants in which ionospheric irregularities were evident.
\end{abstract}

Fabricio dos Santos Prol

fabricioprol@hotmail.com

Paulo de Oliveira Camargo

paulo@fct.unsep.br

1 São Paulo State University - UNESP, Roberto Simonsen, 305, São Paulo, SP 19060-900, Brazil
Keywords Total electron content - Slant TEC · Gridbased tomography · MART · Ionospheric imaging · Ionospheric profiles

\section{Introduction}

The spatial and temporal variation of electron density in the upper atmosphere makes the ionosphere difficult to model. A major difficulty arises in the equatorial region due to the lack of data in the southern hemisphere for use in models such as the international reference ionosphere (IRI) (Bilitza et al. 2011). The global navigation satellite system (GNSS), however, has become an important ally in equatorial ionospheric imaging, since it ensures global coverage. Therefore, the continued availability of new GNSS satellites and denser GNSS networks inspires many international efforts to apply tomographic techniques to reconstruct three-dimensional representations of the ionosphere in the equatorial region (Chartier et al. 2014; Muella et al. 2011; Mridula et al. 2011; Thampi et al. 2004; Franke et al. 2003; Andreeva et al. 2000). In addition, satellite-tosatellite radio occultation (RO) data, such as ionospheric information derived from CHAMP (Wickert et al. 2001) and FORMOSAT-3/COSMIC (Fong et al. 2008) missions, have been found to be a relevant data source in ionospheric tomography for improving the peak height and critical electron density estimation of the ionosphere (Yin and Mitchell 2005) and also for enhancing the vertical resolution of the ionospheric reconstruction (Wen et al. 2007).

A peculiarity of the Brazilian sector is that some ionospheric dynamics over the region are not completely understood. It is affected by plasma bubbles (Ely et al. 2012; Haase et al. 2011), by the equatorial ionization anomaly (EIA) (Nogueira et al. 2013; Tulasi Ram et al. 
2009; Materassi and Mitchell 2005), by being close to the center of the South America Magnetic Anomaly (SAMA) (Abdu et al. 2005) and by layered structures, such as the sporadic E-layer, in the equatorial region (Zhao et al. 2011; Batista et al. 2008; Rishbeth 2000). One of the major topics of investigation is the study of the ionospheric irregularities that occurs at the evening pre-reversal drift, which is believed to be driven by the gravitational Rayleigh-Taylor mechanism, characterized by high vertical gradients of electron density (Abdu 2005). Therefore, tomographic techniques applied to reconstruct a three-dimensional ionosphere might contribute to many studies of ionospheric dynamics over this region.

In general, tomographic algorithms of the ionosphere may be classified into two categories: grid based and function based. The first group uses algebraic techniques for the estimation of the electron density into a grid composed by three-dimensional cells (latitude, longitude, and height) called voxels (Shukla et al. 2010), or two-dimensional cells (pixels) (Yao et al. 2013). Otherwise, functionbased algorithms reduce the ionospheric variations into a set of coefficients (Van de Kamp 2013).

Muella et al. (2011) conducted a pioneer analysis on applying function-based tomographic algorithms in Brazil. However, since this analysis, the Brazilian Network for Continuous GNSS Monitoring (RBMC) has actually grown from 50 to about 100 receivers. This impulse, coupled with the fact that no grid-based techniques had been used to study ionospheric dynamics in Brazil, inspired the use of multiplicative algebraic reconstruction technique (MART) and the existing ground-based GNSS receivers for the recent solar maximum. MART was analyzed for the estimation of critical frequencies of the F2-layer, slant TEC, and ionospheric profiles. The performance of MART was also investigated to reconstruct horizontal representations of EIA and vertical gradients. The main concepts of the tomographic formulation are presented in the next section. In the following section, we summarize our experimental results and then present the conclusions about MART as applied to Brazil.

\section{Tomographic formulation}

The basic quantity required to obtain ionospheric information from GNSS is the total electron content (TEC), which is expressed as the integral of the electron density $n_{e}$ along the path between the GNSS satellite $s$ and the receiving antenna $r$, in a column whose cross-sectional area is equivalent to $1 \mathrm{~m}^{2}$. It can be written as:

$\mathrm{TEC}=\int_{r}^{s} n_{e} \mathrm{~d} s$ where, in computerized ionospheric tomography (CIT), the electron density is the parameter to be reconstructed and the TEC corresponds to the line integral of electron density that slice the ionosphere. For this reconstruction, the ionosphere is broken down into a grid of cells (pixels or voxels) and the TEC may be approximated by a finite sum. Assuming $n_{e j}$ is the electron density for a cell $j$ and $d_{i j}$ the path length of the GNSS signal $i$ inside the boundaries that intersect the cell $j$, Eq. (1) can be formulated as:

$\mathrm{TEC}_{i}=\sum_{j=1}^{J} n_{e j} d_{i j}$

where $j$ ranges from 1 to $J$ (number of voxels in the grid) and $d_{i j}=0$ if the signal does not intercept the corresponding cell.

The path length covered by the GNSS signal in each cell may be calculated from the projection of an ionospheric pierce point (IPP) in each point where the GNSS signal transverses a grid cell (Shukla et al. 2010). Therefore, a reasonable algorithm can be selected to solve this inverse problem and estimate the electron density in each cell using TEC observations.

Due to the incomplete geometrical coverage of the GNSS signals for tomographic applications, an initial estimate for the ionosphere is required, known as a background ionosphere (Bust and Mitchell 2008). The background is usually obtained from empirical models of the ionosphere, such as the IRI, to fill in each cell of the CIT grid. The grid-based algorithm then uses algebraic techniques to distribute the difference between the calculated TEC from the background and the TEC measured by GNSS into the resulting grid.

Algebraic reconstruction technique (ART) was the first algorithm used in computerized tomography (CT). After Austen et al. (1988), different versions of ART were developed for ionospheric imaging, such as the simultaneous algebraic reconstruction technique (SART) (Anderson and Kak 1984) and the MART. While ART and SART are based on a linear formulation to calculate differences between the TEC measured by GNSS and the TEC from the background, MART is based on the following nonlinear iteration (Pryse et al. 1998):

$n_{e j}^{K+1}=n_{e j}^{K}\left(\frac{\mathrm{TEC}_{i}}{\sum_{j=1}^{J} d_{i j} n_{e j}^{K}}\right)^{w d_{i j} / d_{\max }}$

where $n_{e j}^{K+1}$ is the electron density value obtained from iteration $K+1$ and $w$ is a weighting parameter. The $\sum_{j=1}^{J} d_{i j} n_{e j}^{K}$ term is equivalent to a scan through each cell of the ionospheric grid that calculates the TEC value from the background, and $d_{\max }$ is the largest path length of the respective signal $i$. Even when $\sum_{j=1}^{J} d_{i j} n_{e j}^{K}>\mathrm{TEC}_{i}$, MART 
produces nonnegative values, so an advantage of using MART, instead of ART or SART, is the guarantee of nonnegative values for electron density. Many new iterative algorithms are being developed to improve algebraic techniques; however, most of them are based on the principles of ART, SART, or MART (Hobiger et al. 2008; Wen and Liu 2010; Wen et al. 2012).

\section{Experiments, results, and discussion}

A total of 24 days were selected during the solar maximum period in 2013 and 2014. The analysis days were distributed over the four seasons (spring, summer, autumn, winter), covering 3 days for each season in 2013 and another 3 days for each season in 2014. Both years were used to consider behavior of the ionosphere on the differential code bias (DCB) estimation. Table 1 summarizes the days used in the analysis, where the criteria for the selected days is based on the amount of data available from the ionosondes installed in Brazil. A total of four ionosondes were used, located at São Luís $\left(2.3^{\circ} \mathrm{S}, 44^{\circ} \mathrm{W}\right.$; magnetic lat. $\left.0.8^{\circ} \mathrm{S}\right)$, Fortaleza $\left(3.8^{\circ} \mathrm{S}, 38.0^{\circ} \mathrm{W}\right.$; magnetic lat. $\left.5.5^{\circ} \mathrm{S}\right)$, Boa Vista $\left(2.8^{\circ} \mathrm{N}, 60.7^{\circ} \mathrm{W}\right.$; magnetic lat. $\left.12.9^{\circ} \mathrm{N}\right)$, and São José dos Campos $\left(23.21^{\circ} \mathrm{S}, 45.86^{\circ} \mathrm{W}\right.$; magnetic lat. $17.26^{\circ} \mathrm{S}$ ), which enabled a reduction in the critical frequency of the ionospheric F2-layer (foF2) from the ionograms.

All available data from the Brazilian network (RBMC) were used to obtain GNSS observations. GNSS data from the International GNSS Service (IGS) network and from the Low-latitude Ionospheric Sensor Network (LISN) were also included to improve the geometrical coverage of the GNSS signals. The locations of the GNSS receivers and the ionosondes are presented in Fig. 1.

All the experiments were executed with the following processing settings: (1) GNSS observations of code (C1, P2) and phase from $L_{1}$ and $L_{2}$ bands were made; (2) TEC was estimated using the leveled carrier phase ionospheric observable (Ciraolo et al. 2007); (3) the DCB was previously estimated for each day by using VTEC maps

Table 1 Days selected for analysis

\begin{tabular}{lllll}
\hline Year & Summer & Autumn & Winter & Spring \\
\hline 2013 & Jan 25 & May 15 & Aug 15 & Nov 5 \\
& Feb 2 & May 18 & Aug 21 & Nov 6 \\
& Feb 3 & May 19 & Aug 22 & Nov 25 \\
2014 & Jan 5 & May 14 & Jul 25 & Oct 3 \\
& Feb 15 & May 19 & Jul 26 & Oct 16 \\
& Feb 20 & May 22 & Jul 28 & Nov 1 \\
\hline
\end{tabular}

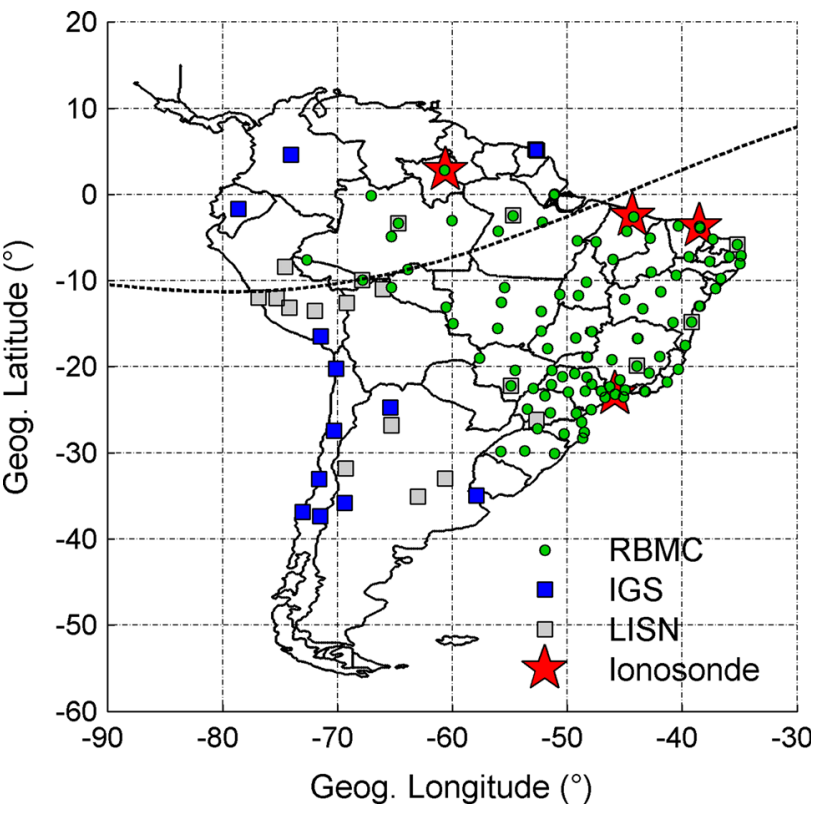

Fig. 1 Location of the GNSS receivers and ionosondes used

available from IGS (Prol and Camargo 2014); (4) cycle slips were detected by using a recursive method, based on widelane ambiguity (Blewitt 1990); (5) IGS precise ephemerides described the satellite positions from GPS and GLONASS constellations for an elevation mask of $20^{\circ}$; (6) the MART algorithm was applied on a voxel-based grid with a resolution of $4^{\circ} \times 4^{\circ} \times 10 \mathrm{~km}$ in latitude, longitude, and height; and (7) the background ionosphere and the plasmasphere correction was calculated by the IRI routines.

The first analysis was performed to detect the accuracy of the MART algorithm on the estimation of the foF 2 . The second was done to check the agreement of the TEC estimated by tomography with the TEC retrieved from the GNSS observations. Therefore, a comparison between ionospheric profiles obtained from the MART algorithm, IRI model, and ionosondes is presented. Finally, ionosphere representations generated by MART are analyzed.

\section{Critical frequency}

In the experiment to estimate the critical frequency, reference values were obtained through the ionosondes. MART and the IRI model provided an estimated critical frequency for every fifteen minutes, and at instants when it was not possible to obtain the foF 2 from the ionograms, a linear interpolation was performed to make the 15-min intervals consistent. Figure 2 shows the average critical frequency estimated by MART and observed from the ionosondes located at Boa Vista (BV), São José dos Campos (SJC), Fortaleza (Fort), and São Luís (SL). 

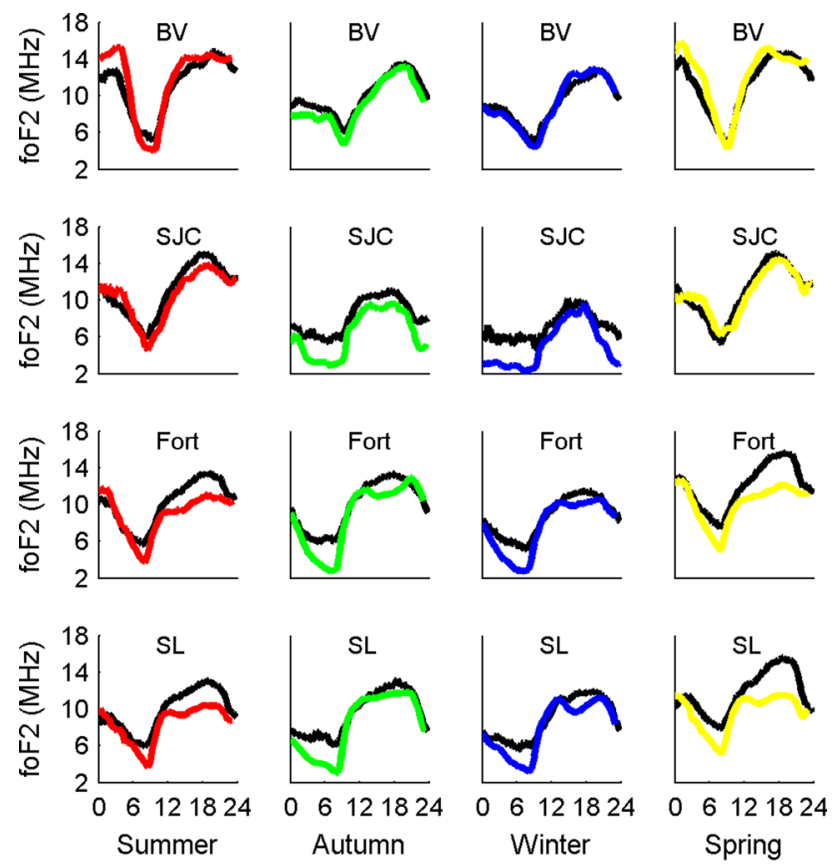

Hour (UT)

Fig. 2 Mean values of the critical frequency with MART (black line) and ionosondes

Figure 3 shows the root-mean-square error (RMSE) results for MART and IRI.

At the Boa Vista location, a total RMSE equal to $1.09 \mathrm{MHz}$ was calculated for MART and $1.43 \mathrm{MHz}$ for IRI. At SJC, the critical frequency estimation showed a total RMSE equal to $1.69 \mathrm{MHz}$ for MART and $1.48 \mathrm{MHz}$ for IRI. On the other hand, a superior total RMSE for MART was calculated at Fortaleza and São Luis, corresponding to 1.77 and $1.89 \mathrm{MHz}$, respectively, for MART and 1.57 and $1.28 \mathrm{MHz}$, respectively, for IRI. Since the IRI model was used as background, the foF2 estimation and error were similar for MART and IRI, but significant discrepancies and errors were checked.

We found that the lowest RMSE values for MART were obtained at Boa Vista and SJC; however, higher discrepancies between MART and IRI were calculated at Fortaleza and São Luís. These discrepancies were more evident during spring and summer in the daytime, between 12 and 20 hours universal time (UT), corresponding to 09 and 17 hours local time (LT), where MART showed higher values of RMSE. Thus, MART had a worse performance during periods with high values of foF2 at Fortaleza and São Luís. This mainly happened due to the distribution of the GNSS stations near to these ionosondes. Fortaleza and São Luís are located close to the edge of the reconstructed area, and the electron density estimation was affected due to incomplete geometrical coverage by the GNSS receivers. In those cases, GNSS signals from the Atlantic Ocean were not used, degrading the geometry at the edge of the
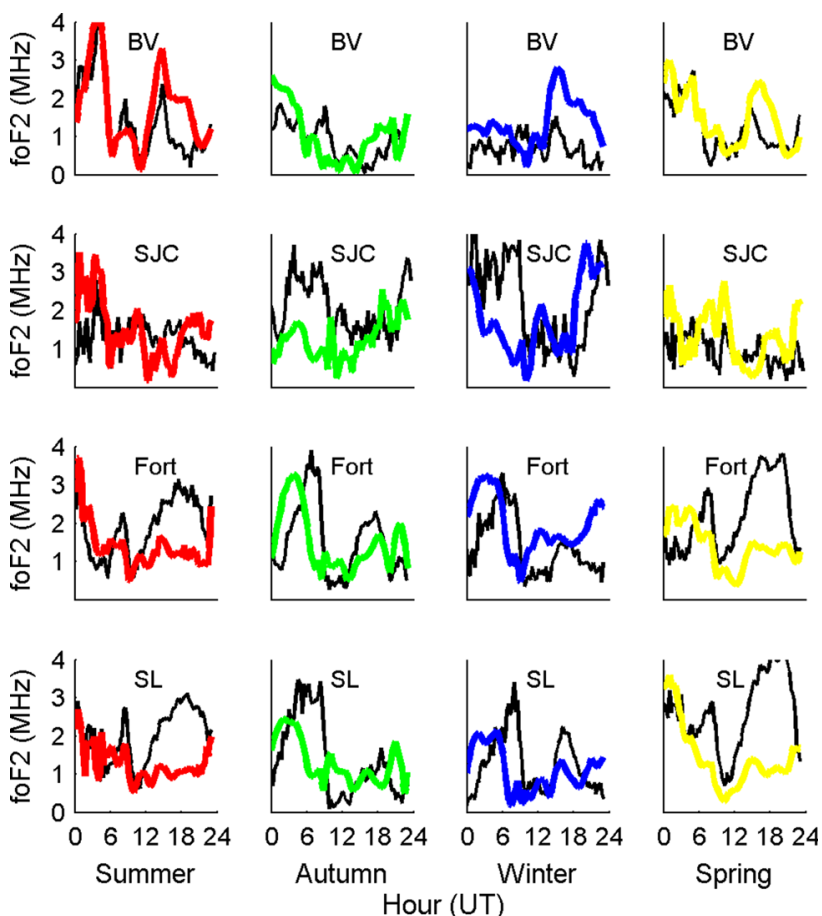

Fig. 3 RMSE values of the critical frequency with MART (black line) and IRI

reconstructed area because no GNSS stations were located offshore, close to the Brazilian sector.

Furthermore, greater RMSE values in SJC, Fortaleza, and São Luis were obtained in the nighttime between 00 and 09 hours UT for winter and autumn. It is possible to see, at the same instants in Fig. 2, larger discrepancies between MART and the ionosondes, where MART overestimated the critical frequency. This overestimation occurred mainly due to the TEC estimation, which considered both ionosphere and plasmasphere electron densities. Despite the plasmasphere correction using IRI, the IRI model was not completely successful in the TEC plasmasphere estimation, mainly because the contribution of the plasmasphere to TEC is greater in winter and autumn, as is shown in Balan et al. (2002) and Jin et al. (2007). Due to the geometry limitation on the CIT, the electron density was then overestimated mostly in the peak height of the ionosphere. Even more, some of these high RMSE values for winter and autumn corresponded to the local sunrise, when the sunlight first reaches the upper atmosphere. So the TEC increased before the critical frequency has increases and then the tomographic reconstruction overestimates the foF2.

\section{Total electron content}

Here the GNSS station BRAZ $\left(15.93^{\circ} \mathrm{S}, 47.86^{\circ} \mathrm{W}\right)$ was used to provide reference values for TEC, since it is located 
in the center of Brazil and is affected by the southern crest of EIA. The DCB having been previously estimated, it was possible to extract the Slant TEC (STEC) from GNSS observations and hence compare the STEC estimated by MART with the STEC calculated using the IONosphere map EXchange format (IONEX) files provided from Centre for Orbit Determination in Europe (CODE). The conversion of the VTEC obtained from CODE maps to STEC was done using the standard geometric mapping function.

Figure 4 shows the RMSE calculated for all the 24 days analyzed, where the GNSS observations from BRAZ were used on the tomographic reconstruction and also on the IONEX production. The STEC values were estimated every $15 \mathrm{~min}$, and the MART RMSE was lower than the IONEX RMSE for almost all instants. The total RMSE was 6.5 TEC Units (TECU) and 8.9 TECU for MART and CODE, respectively, showing a better tomographic performance. Besides that, the STEC with IRI routines was also calculated, but due to the high values of the RMSE, with a total RMSE equal to 17.2 TECU, this result is not shown in Fig. 4. A similar agreement in the RMSE is noticed for MART and CODE maps, but with a principal RMSE difference for 20 hours UT (17 hours LT) and 04 hours UT (01 hours LT), where the MART algorithm showed lower RMSE. These instants correspond to the evening pre-reverse period, when the ionospheric irregularities are more effective to impact the GNSS observations.

An example of the STEC estimated with MART and calculated with the CODE maps at instants with ionospheric irregularities is presented in Fig. 5. This figure presents results for the satellite with the pseudorandom number (PRN) one from GPS on the day Feb 20, 2014, where the MART algorithm was better at representing the hourly variations than the CODE maps.
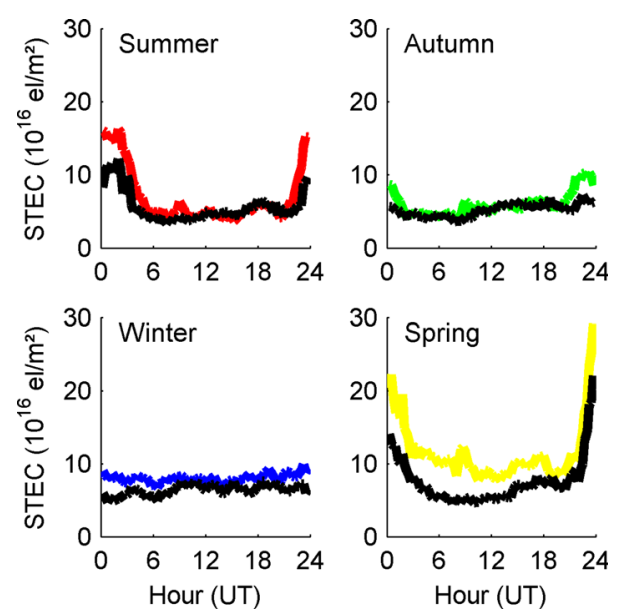

Fig. 4 RMSE estimation with MART (black lines) and calculated with CODE IONEX maps at station BRAZ

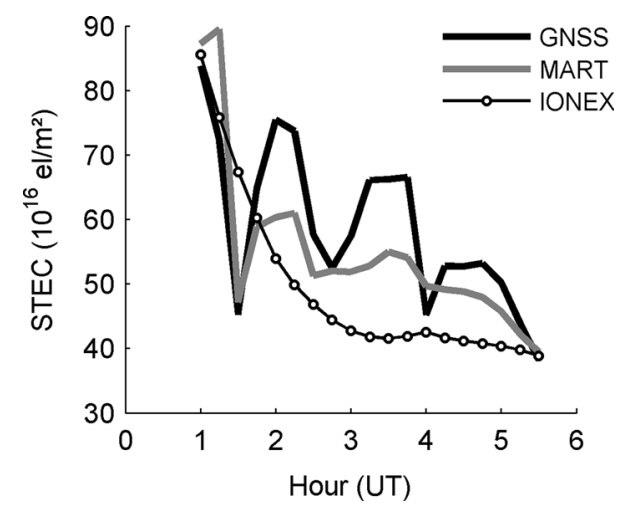

Fig. 5 STEC estimation at instants with ionospheric irregularities

\section{Ionospheric profiles}

Profiles from the ionosonde located at Boa Vista were constructed using the following parameters reduced from ionograms: critical frequency and peak height of F2-layer (foF2, hmF2), F1-layer (foF1, hmF1), and E-layer (foE, hmE), bottomside thickness (B0) and shape (B1), and propagation factor $\mathrm{M}(3000) \mathrm{F} 2$. These parameters in conjunction with IRI functions were used to construct the ionosonde profiles. It was possible this way to calculate values of the ionospheric reflection frequency in altitudes above the peak height. Mean profiles were then constructed at each hour for all the days and are presented in Fig. 6. When the ionosonde data were not available, a linear interpolation was carried out.

In general, the MART algorithm showed the daily variation of the electron density. When MART estimated higher frequency values than IRI, the ionosonde also indicated higher values than IRI. The inverse was also verified; when MART showed lower electron densities than IRI, ionosonde profiles also showed lower values than IRI. However, when both ionosonde and MART presented higher frequencies than IRI, the MART algorithm overestimated the frequency values, mainly in the peak height of the profiles. This occurred mostly because the ionosonde does not make measurements at higher altitudes than the peak height, while TEC considers the electron density for the entire GNSS signal path. Increased electron density at the higher altitudes of the ionosphere coupled with incomplete geometric coverage of GNSS for tomographic applications provided an overestimation of the ionospheric electron density in the peak heights.

Furthermore, higher discrepancies between the profiles were calculated between 21 and 01 hours UT (18 and 22 hours LT). These principal discrepancies occurred due to vertical drift during the dusk period, elevating the ionosphere to higher altitudes. The IRI model did not provide a sufficiently accurate estimation for the peak altitude of the ionosphere in the pre-reverse period, and the 

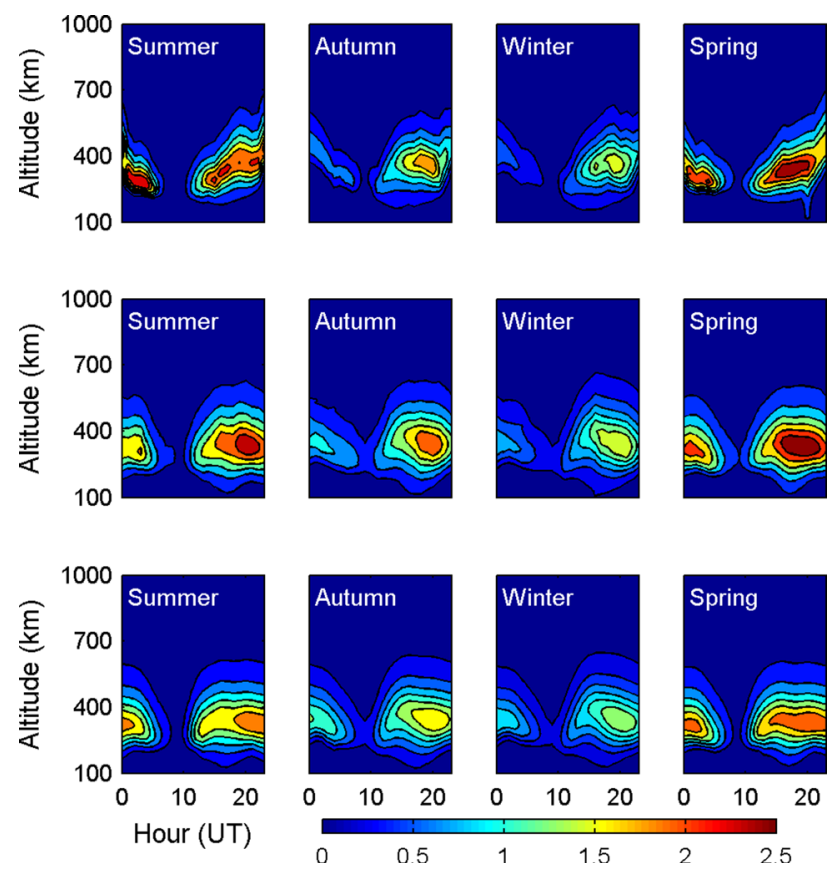

Fig. 6 Mean ionospheric profiles for the ionosonde, MART, and IRI (Boa Vista). The top, middle, and bottom panels show the ionospheric profiles obtained from ionosonde, MART, and IRI, respectively. The unit of the color bar is $10^{12} \mathrm{el} / \mathrm{m}^{3}$

IRI peak height was used as background in the tomographic reconstruction. However, due to the limitations of the CIT system, the peak height was estimated by MART with similar accuracy as the IRI model.

\section{Ionospheric representations in Brazil}

Figure 7 presents average VTEC maps constructed with MART in order to view the horizontal behavior of the ionosphere for the different seasons of the years. In addition, Fig. 8 shows average representations of the ionospheric profiles, aiming to visualize the vertical variations of the ionosphere over Brazil. The longitudinal sector of $50^{\circ} \mathrm{W}$ was used, since it has one of the highest numbers of GNSS receivers for different latitudes, and because it enables analysis of EIA. Both figures show average maps for all the days used in the experiments and for 3 hours: The first $(00$ UT) shows instants with the intensification of EIA due to vertical drift in the evening pre-reverse period; the second (12 UT) shows instants at dawn; and the last hour (18 UT) shows instants during the maximum daily values of VTEC.

In Figs. 7 and 8, it is possible to see the EIA intensification due to the vertical drift of the ionosphere to higher altitudes. The peak height at $00 \mathrm{UT}$ in the spring and summer is higher than in winter and autumn, and actually the EIA presented higher values of VTEC for spring and summer. In addition, at 00 UT the E-layer and F1-layer are not represented on the profiles, increasing the vertical gradients of electron density
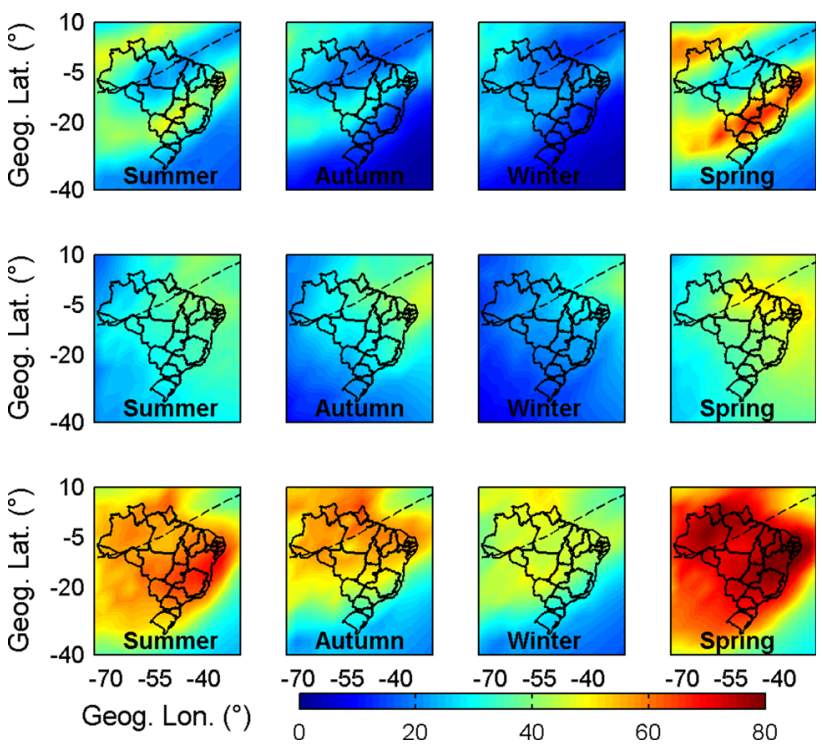

Fig. 7 Mean VTEC ionospheric maps. The top, middle, and bottom panels show the ionospheric VTEC maps obtained at 00, 12, and 18 hours UT, respectively. The unit of the color bar is $10^{16} \mathrm{el} / \mathrm{m}^{2}$
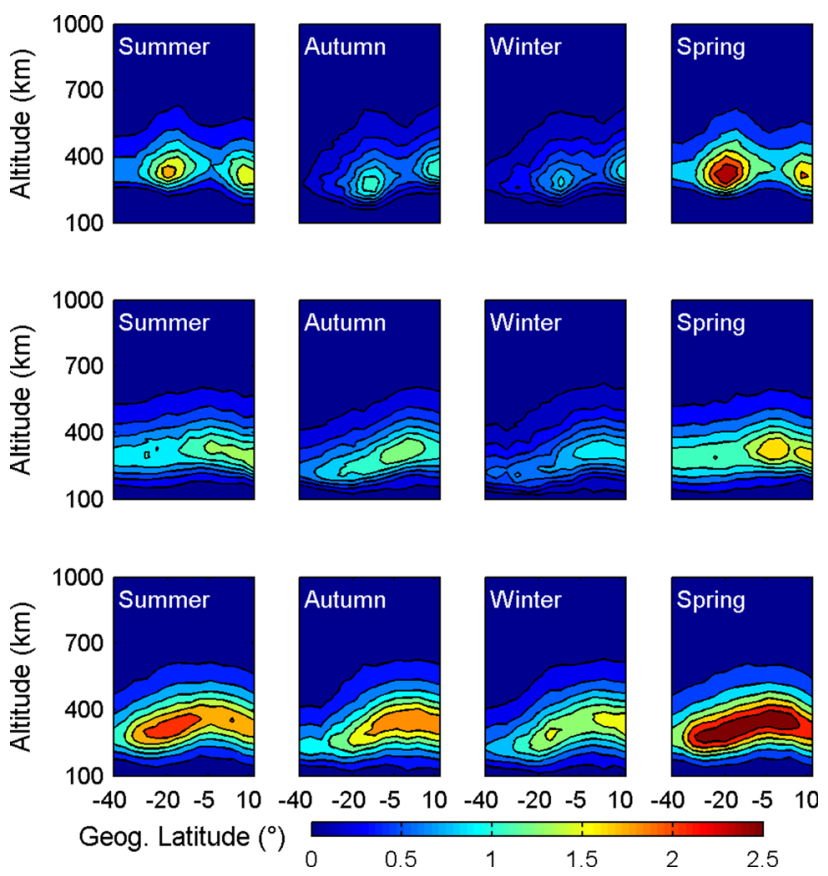

Fig. 8 Mean ionospheric profiles for the $50^{\circ} \mathrm{W}$ sector. The top, middle, and bottom panels show the ionospheric profiles obtained at 00,12 , and 18 hours UT, respectively. The unit of the color bar is $10^{12} \mathrm{el} / \mathrm{m}^{3}$

with the altitudes. Although 18 UT marks the maximum in VTEC values, the profiles showed smaller electron density vertical gradients, in comparison with $00 \mathrm{UT}$, mainly due to the absence of the E-layer and F1-layer. Thus, these average ionospheric profiles show the pre-conditions favorable to the development of instability in the plasma for the spring and 
summer at $00 \mathrm{UT}$, where a heavy fluid is supported by a light fluid, indicating similar characteristics to the RayleighTaylor instability. These instants are also correlated with the hours when ionospheric irregularities were checked for the STEC estimative.

Also, the higher values of VTEC were estimated during the spring. The spring marks the passage of the sun to the southern hemisphere and, in comparison with summer, showed higher solar activity (the largest sunspot of the solar cycle 24 was detected during October, 2014). In this way, EIA during the spring was more intense. It is also possible to check asymmetry on the EIA crest due to the seasonal variations in Figs. 7 and 8 . When the sun is above the southern hemisphere, the southern crest is located at higher altitudes and with higher VTEC values. On the other hand, when the sun is above the northern hemisphere, the northern crest is represented at higher altitudes and with greater VTEC values.

\section{Summary and conclusions}

GNSS receivers from RBMC, IGS, and LISN, coupled with four ionosondes installed in Brazil were used to evaluate the MART algorithm during the recent solar maximum period. The RMSE of fof 2 for MART showed similar agreement to the RMSE obtained from IRI for two ionosondes (Boa Vista and São José dos Campos). In the other ionosondes (Fortaleza and São Luís), the tomographic reconstruction was affected by the inadequate distribution of the GNSS receivers on the vicinity of ionosondes. In these locations, the geometrical coverage of the GNSS signals was not completely suitable for tomographic application because they are located close to the edge of the reconstructed area, and there were no GNSS receivers for IGS, RBMC, or LINS, over the Atlantic Ocean near the Brazilian sector.

We presented a comparison between ionospheric profiles constructed with MART, IRI, and ionosonde. The daily variations were better represented using MART, in comparison with the IRI model, but with overestimated values of electron density. This occurred because the ionosonde does not observe the ionosphere in altitudes above the peak height, while TEC considers the electron density over the entire GNSS signal path from the satellite to the receiving antenna. In addition, the peak height of the ionospheric profiles from MART was similar to those estimated by IRI, used as background ionosphere for the tomography, and this contributed to the overestimated values at the peak height. Moreover, the results presented an overestimation of the electron density due to the plasmasphere. The plasmasphere correction was calculated using the IRI model, but this was not completely accurate for the tomographic application. An overestimation in the electron density then occurred at nighttime in winter and autumn.
The MART algorithm presented a better performance on the STEC estimation in comparison with the STEC calculated with IONEX maps from CODE. The total RMSE calculated at station BRAZ was 6.5 TECU for MART and 8.9 TECU for the IONEX maps. Furthermore, average ionospheric profiles obtained from the MART algorithm showed high vertical gradients of electron density during the current solar maximum period for the spring and summer. These conditions were favorable for the development of instability in the plasma at the evening pre-reverse period and irregularities on the STEC estimation were checked at the same instants.

Therefore, the analyses suggest that MART can be used to combine GNSS-TEC observations with the IRI model to construct three-dimensional ionospheric representations and then study the ionospheric dynamics in Brazil. MART enabled constructing ionospheric VTEC maps and vertical profiles to show daily variations of the ionosphere, making it possible to analyze the intensification of the EIA and correlate this information with the vertical drift that occurs over the equatorial ionosphere during the pre-reverse period. But it should be taken into account that representation of the vertical drift altitude is inherent to the ionospheric background used to perform algebraic techniques.

Acknowledgments This work was undertaken on grants of the master degree scholarship from CAPES (Coordenação de Aperfeiçoamento de Pessoal de Nível Superior) and scholarship research productivity of $\mathrm{CNPq}$ (Conselho Nacional de Pesquisa). The authors are grateful to Instituto Brasileiro de Geografia e Estatística (IBGE), International GNSS service (IGS) and Low-latitude Ionospheric Sensor Network (LISN) project for providing dual-frequency GNSS receivers. We are also grateful to the Instituto Nacional de Pesquisas Espaciais (INPE) and Universidade do Vale do Paraíba (UNIVAP) for providing ionosondes installed in Brazil.

\section{References}

Abdu MA (2005) Equatorial ionosphere-thermosphere system: electrodynamics and irregularities. Adv Space Res 35(5):771-787

Abdu MA, Batista IS, Carrasco AJ, Brum CGM (2005) South Atlantic magnetic anomaly ionization: a review and a new focus on electrodynamics effects in the equatorial ionosphere. J Atmos Sol Terr Phys 67:1643-1657

Anderson AH, Kak AC (1984) Simultaneous algebraic reconstruction technique (SART): a superior implementation of the ART algorithm. Ultraso Imaging 6:81-94

Andreeva ES, Franke SJ, Yeh KC, Kunitsyn VE (2000) Some features of the equatorial anomaly revealed by ionospheric tomography. Geophys Res Lett 27:2465-2468

Austen JR, Franke SJ, Liu CH (1988) Ionospheric imaging using computerized tomography. Radio Sci 23:299-307

Balan N, Otsuka Y, Tsugawa T, Miyazaki S, Ogawa T, Shiokawa K (2002) Plasmaspheric electron content in the GPS ray paths over Japan under magnetically quiet conditions at high solar activity. Earth Planets Space 54:71-79

Batista IS, Abdu MA, Carrasco AJ, Reinisch BW, de Paula ER, Schuch NJ, Bertoni F (2008) Equatorial spread F and sporadic 
E-layer connections during the Brazilian Conjugate Point Equatorial Experiment (COPEX). J Atmos Sol Terr Phys 70:1133-1143

Bilitza D, McKinnel LA, Reinisch B, Fuller-Rowell T (2011) The international reference ionosphere today and in the future. J Geod 85(12):909-920

Blewitt G (1990) An automated editing algorithm for GPS data. Geophys Res Lett 17(3):199-202

Bust GS, Mitchell CN (2008) History, current state, and future directions of ionospheric imaging. Rev Geophys 46:RG1003

Chartier AT et al (2014) Ionospheric imaging in Africa. Radio Sci 49:19-27

Ciraolo L, Azpilicueta F, Brunini C, Meza A, Radicella SM (2007) Calibration errors on experimental slant total electron content (TEC) determined with GPS. J Geod 81(2):111-120

Ely CV, Batista IS, Abdu MA (2012) Radio occultation electron density profiles from the FORMOSAT-3/COSMIC satellites over the Brazilian region: a comparison with Digisonde data. Adv Space Res 49(11):1553-1562

Fong CJ, Yang SK, Chu CH, Huang CY, Yeh JJ, Lin CT, Kuo TC, Liu TY, Yen NL, Chen SS, Kuo YH, Liou YA, Chi S (2008) FORMOSAT-3/COSMIC constellation spacecraft system performance: after 1 year in orbit. IEEE Trans Geosci Remote Sens 46:3380-3394

Franke SJ, Yeh KC, Andreeva ES, Kunitsyn VE (2003) A study of the equatorial anomaly ionosphere using tomographic images. Radio Sci 38(1): 1011

Haase JS, Dautermann T, Taylor MJ, Chapagain N, Calais E, Pautet D (2011) Propagation of plasma bubbles observed in Brazil from GPS and airglow data. Adv Space Res 47:1758-1776

Hobiger T, Kondo T, Koyama Y (2008) Constrained simultaneous algebraic reconstruction technique (C-SART) - a new and simple algorithm applied to ionospheric tomography. Earth Planets Space 60(2):727-735

Jin S, Cho JH, Park JU (2007) Ionospheric slab thickness and its seasonal variations observed by GPS. J Atmos Sol Terr Phys 69(15): 1864-1870

Materassi M, Mitchell CN (2005) Imaging of the equatorial ionosphere. Ann Geophys 48(3):477-482

Mridula N, Manju G, Pant TK, Ravindran S, Jose L, Alex S (2011) On the significant impact of the moderate geomagnetic disturbance of March 2008 on the equatorial ionization anomaly region over Indian longitudes. J Geophys Res 116:A07312

Muella MTAH, de Paula ER, Mitchell CN, Kintner PM, Paes RR, Batista IS (2011) Tomographic imaging of the equatorial and low-latitude ionosphere over central-eastern Brazil. Earth Planets Space 63:129-138

Nogueira PAB, Abdu MA, Souza JR, Batista IS, Bailey GJ, Santos AM, Takahashi H (2013) Equatorial ionization anomaly development as studied by GPS TEC and foF2 over Brazil: a comparison of observations with model results from SUPIM and IRI-2012. J Atmos Sol Terr Phys 104:45-54

Prol FS, Camargo PO (2014) Estimativa da tendência diferencial do código nos receptores GNSS. Bol Ciênc Geod 20(4):735-749

Pryse SE, Kersley L, Mitchell CN, Spencer PSJ, Williams MJ (1998) A comparison of reconstruction techniques used in ionospheric tomography. Radio Sci 33(6):1767-1779

Rishbeth H (2000) The equatorial F-layer: progress and puzzles. Ann Geophys 18:730-739

Shukla AK, Sivaraman MR, Bandyopadhyay K (2010) A comparison study of voxel based multi and two-layer tomography models over Indian region using GPS data. Int $J$ Remote Sens 31(10):2535-2549

Thampi SV, Pant TK, Ravindran S, Devasia CV, Sridharan R (2004) Simulation studies on the tomographic reconstruction of the equatorial and low-latitude ionosphere in the context of the Indian tomography experiment: CRABEX. Ann Geophys 22:3445-3460

Tulasi Ram S, Su SY, Liu CH (2009) FORMOSAT-3/COSMIC observations of seasonal and longitudinal variations of equatorial ionization anomaly and its interhemispheric asymmetry during the solar minimum period. J Geophys Res 114:A06311

Van de Kamp MMJL (2013) Medium-scale 4-D ionospheric tomography using a dense GPS network. Ann Geophys 31:75-89

Wen DB, Liu SZ (2010) A new ionospheric tomographic algorithmconstrained multiplicative algebraic reconstruction technique (CMART). J Earth Syst Sci 119(4):489-496

Wen DB, Yuan YB, Ou JK, Huo XL, Zhang KF (2007) Threedimensional ionospheric tomography by an improved algebraic reconstruction technique. GPS Solut 11(4):251-258

Wen DB, Wang Y, Norman R (2012) A new two-step algorithm for ionospheric tomography solution. GPS Solut 16(1):89-94

Wickert J, Reigber C, Beyerle G, König R, Marquardt C, Schmidt T, Grunwaldt L, Galas R, Meehan TK, Melbourne WG, Hocke K (2001) Atmosphere sounding by GPS radio occultation: first results from CHAMP. Geophys Res Lett 28(17):3263-3266

Yao YB, Chen P, Zhang S, Chen JJ (2013) A new ionospheric tomography model combining pixel-based and function-based models. Adv Space Res 52:614-621

Yin P, Mitchell CN (2005) Use of radio-occultation data for ionospheric imaging during the April 2002 disturbances. GPS Solut 9:156-163

Zhao B, Wan W, Yue X, Liu L, Ren Z, He M, Liu J (2011) Global characteristics of occurrence of an additional layer in the ionosphere observed by COSMIC/FORMOSAT-3. Geophys Res Lett 38:L02101

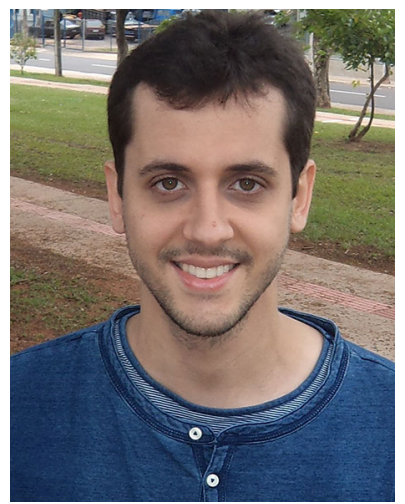

Fabricio dos Santos Prol received his BE degree in Cartographic Engineering in 2011 and MSc degree in Cartographic Sciences in 2015 from Universidade Estadual Paulista (UNESP) at Presidente Prudente, SP, Brazil. He is a $\mathrm{PhD}$ candidate at UNESP, and the focus of his current research lies in ionospheric modeling using data assimilation.

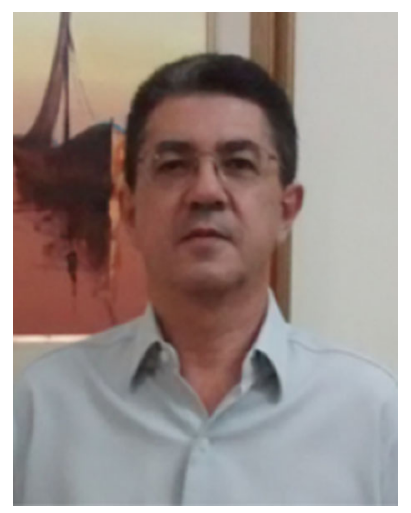

Paulo de Oliveira Camargo received his $\mathrm{BE}$ degree in Cartographic Engineering in 1985 from Universidade Estadual Paulista (UNESP), his MSc degree in 1992 and $\mathrm{PhD}$ degree in 1999 in Geodetic Sciences from the Universidade Federal do Paraná (UFPR), Brazil. He is professor of the graduate and postgraduate program at UNESP focusing on the following topics: GNSS, ionosphere, quality control, and least-squares adjustment. 